\title{
Fracture risk in Systemic Lupus Erythematosus patients over 28 years
}

Daniela Garelick ${ }^{1}$, Sara Moreira Pinto ${ }^{2}$, Filipa Farinha ${ }^{3}$, Tatiana Pires ${ }^{4}$, Emon Khan ${ }^{3}$, David Isenberg ${ }^{3}$

${ }^{1}$ Rheumatology Department, Sheba Medical Center, Ramat Gan, Israel Internal Medicine Department, ${ }^{2}$ Pedro Hispano Hospital, Porto, Portugal; ${ }^{3}$ Rheumatology Department, University College London Hospital, London, United Kingdom; ${ }^{4}$ Serviço de Medicina 1, Hospital de Santo André, Centro Hospitalar de Leiria, Leiria, Portugal

\section{Corresponding Author:}

Professor David Isenberg, Room 424, The Rayne Institute, 5 University Street, London WC1E 6JF d.isenberg@ucl.ac.uk

\section{Acknowledgements and funding:}

Professor Isenberg gratefully acknowledges the support of the Biomedical Centre award to University College London and University College Hospital

FF was funded by LUPUS UK (Grant 1543411)

\section{Disclosures:}

Authors declare no conflicts of interest

\section{Word count}

Abstract: 238

Main text: 3044 


\section{Abstract}

Background: Chronic glucocorticoid use is complicated by osteoporosis and increases the risk of fragility fractures. EULAR guidelines on SLE management recommend reducing chronic glucocorticoid dosage to $\leq 7.5 \mathrm{mg} /$ day to minimise this risk. We examined the relationship of glucocorticoid dose on fragility fracture risk in a cohort of SLE patients.

Methods: Retrospective analysis of SLE patients attending University College Hospital over 28 years was undertaken. Collected data included consecutive steroid dose, DEXA scans and fragility fractures.

Results: We collected data on 250 patients with a median of 17 years' follow-up. Fragility fractures were diagnosed in 28 (11.2\%) patients and the mean age of first fracture was $51 \pm 16$ years. 94\% received glucocorticoids, the average dose being $6.20 \mathrm{mg} /$ day. Patients with fragility fractures had a lower average daily dose (5.36 versus $6.23 \mathrm{mg} /$ day) but a higher median cumulative dose $(25.19 \mathrm{~g}$ versus $20.96 \mathrm{~g})$. These differences were not significant $(\mathrm{p}=0.127$ and 0.229 , respectively). $93 \%$ of patients received vitamin $\mathrm{D}$ and $85 \%$ received calcium. Cox regression analysis showed older age at SLE diagnosis, osteoporosis and secondary hyperparathyroidism were associated with fragility fractures. Glucocorticoid dose was not significantly associated with the occurrence of fragility fractures. Twenty-two patients with fractures were treated with bisphosphonates, two with denosumab and two with teriparatide.

Conclusions: We found no significant association between glucocorticoid treatment and fragility fractures in our group of patients, however, a prospective study including more patients not treated with corticosteroids, would be necessary to confirm these results.

Keywords: Systemic lupus erythematous, glucocorticoids, osteoporosis and fractures

\section{Key messages}

- Average daily or cumulative dosing of glucocorticoids were not associated with increased osteoporosis or fractures

- Glucocorticoid prescribing practice in our clinic has not changed during 28-years' follow-up in this study 


\section{Introduction}

It has long been recognised that glucocorticoids are associated with a constellation of metabolic sideeffects including osteoporosis, which increases the risk of fragility fractures. This negative impact on the skeleton is dose and duration dependent with higher doses having a greater impact[1]. Glucocorticoids alter bone remodelling by decreasing the rate of bone formation and increasing the rate of bone resorption, reducing bone mineral density (BMD) thereby increasing the risk of fragility fractures.

Glucocorticoids decrease the rate of bone formation by promoting the increase in regulators of osteoblast differentiation and survival, dickkopf-1 and sclerostin, leading to increased levels of nuclear factor-kB. The consequences are decreased osteoblast proliferation and maturation as well as increased osteoblast apoptosis[2]. Osteoclast activity and number are also increased by glucocorticoids. This is the result of several actions: increased production of macrophage colony stimulating factor and receptor activator of nuclear factor-кB ligand, whilst suppressing osteoprotegerin production by osteoblasts and osteocytes[3]. These changes are associated with an early, but transient, increase in bone resorption. These adverse effects usually develop rapidly after the initiation of glucocorticoid treatment. The effects, however, significantly decrease soon after treatment cessation[4].

Glucocorticoid treatment remains the most common cause of secondary osteoporosis. Treatment with glucocorticoids remains widely used in both acute and chronic management of systemic lupus erythematosus (SLE). The 2019 update of the European League Against Rheumatism (EULAR) guidelines for the management of SLE recommend minimising the use of glucocorticoids[5]. The aim is a daily dose $\leq 7.5 \mathrm{mg} /$ day, as higher doses are associated with a significantly increased risk of irreversible organ damage including fractures[6].

Several studies have found an increased prevalence of fragility fractures and reduced BMD in patients with SLE compared to the general population[7-10]. Reports of the incidence of osteoporosis in patients with SLE vary from 1.4-68\%[11]. A recent large cohort-based study found that patients with SLE have a two-fold increase in fragility fractures versus matched comparators and this risk was only mildly reduced when corrected for glucocorticoid use[12]. Another cohort study in the US showed a five-fold increase in fracture occurrence in women with SLE patients compared to the general population of women[13]. Overall, fragility fractures are more common in SLE patients and the cause is multifactorial. 
Given the harmful effects of glucocorticoids and more stringent guidelines regarding steroid dosing, we examined a group of 250 patients from the University College Hospital SLE cohort on whom we had detailed information about their bone health. These patients have been followed up for 28 years. The aim was to assess the impact of steroid dosing on the risk of osteoporosis and fragility fractures in our SLE population. Furthermore, we wanted to assess whether there had been a change in the pattern of glucocorticoid prescribing over time, in particular, leading to a reduction in average daily dosage for our cohort of patients.

\section{Patients and Methods}

Data source and study design:

We conducted a retrospective study of patients under the care of the SLE clinic at University College Hospital for over 28 years. Patients were included if they were over the age of 18 , had a diagnosis of SLE (that was consistent with revised criteria of the American College of Rheumatology (14)), had undergone at least one dual-energy $x$-ray absorptiometry (DEXA) scan and with clinic follow-up that documented medical treatment, including patients receiving glucocorticoids and those who did not.

DEXA scan results for the neck of femur and lumbar spine were collected for all the patients. Consecutive glucocorticoid dosing from each clinic visit, where applicable, was also collected. Demographic data were collected, as well as data on comorbidities and medications for the prevention of or treatment for osteoporosis were also collected.

Data were collected following interrogation of paper and electronic patient records. Contemporaneous clinical notes and clinic letters for all the patients were reviewed for the whole duration of their care.

\section{Covariates}

Race/ethnicity was categorized as Black African or Caribbean, Caucasian, South Asian and Other. Data were collected regarding smoking status, concurrent rheumatoid arthritis /juvenile idiopathic arthritis, vitamin $\mathrm{D}$ deficiency, end-stage renal disease (ESRD), primary or secondary hyperparathyroidism, hyperthyroidism and gonadal failure. Data on treatment with vitamin $D$, calcium supplementation, bisphosphonates, denosumab, teriparatide, raloxifene, oestrogen and strontium ranelate was also collected from contemporaneous clinical records made by the treating physician. 
Cumulative glucocorticoid dosing was calculated using the daily dosing recorded at every clinic visit and multiplying it by the duration of treatment for each dose. The average daily dose was calculated by dividing the cumulative dose by the total period that the patient was recorded to be treated with glucocorticoids. All the glucocorticoid calculations are expressed as prednisolone equivalents. Fragility fractures were defined as that recorded by the treating physician in contemporaneous notes and included neck of femur, neck of humerus, distal radius and vertebral fractures.

Statistical analysis

The data were analysed using SPSS $^{\circledR}$ software v22; values $p<0,05$ were considered statistically significant. Categorical variables were compared using Pearson's Chi-Square or Fisher's exact test; Mann-Whitney $U$ test was used for numerical variables. We performed Cox regression analysis to investigate predictors of fractures, osteoporosis and osteopenia. Kaplan-Meier curves were obtained.

\section{Results}

We identified 250 patients with a diagnosis of SLE and extensive data regarding DEXA scanning and consecutive medication follow-up. These patients were seen in University College Hospital SLE clinic between 1990 and 2018. Patient characteristics are shown in Table 1. The majority of patients were female (92\%) and Caucasian (52\%), and the mean age at diagnosis was 27 years (ranging from $9-75$ years). $5 \%$ had concurrent rheumatoid arthritis. On reviewing the risk factors for osteoporosis; less than a third of the patients (27\%) were smokers, mean BMI was $24 \mathrm{~kg} / \mathrm{m}^{2}$, ESRD was diagnosed in $3 \%$ of patients, secondary hyperparathyroidism in $2 \%$, also gonadal failure in $2 \%$, hyperthyroidism in $1 \%$, and $11 \%$ of patients had been diagnosed with vitamin $D$ deficiency. The majority of patients were treated with vitamin D supplementation (93\%) and a high proportion was also treated with calcium supplementation (85\%). Bisphosphonates were prescribed to a third of patients with many fewer receiving hormone replacement therapy $(2 \%)$ and only $1 \%$ of patients receiving any of the following: denosumab, teriparatide, strontium ranelate or raloxifene. In our cohort, $94 \%$ of the patients were treated with glucocorticoids at some point during their follow up. The average daily dose of prednisolone was $6.20 \mathrm{mg} /$ day and the median cumulative dose of prednisolone was $21.68 \mathrm{~g}$. We did not include the patients that were not treated with glucocorticoids in these calculations.

Fracture incidence 
Fragility fractures were diagnosed in 28 patients (11.2\%). When comparing the group of patients with fractures versus those without, there was no significant difference in the average daily glucocorticoid dose or cumulative dose. There were some differences in clinical characteristics between the groups; those with fractures were older at SLE diagnosis (median age 36 years versus 27 years, $p=0.009$ ), there was a significantly higher proportion of patients with osteoporosis noted in the group with fractures than those without ( $36 \%$ versus $14 \%, p=0.006$ ) and an increased proportion of patients with secondary hyperparathyroidism were in the group with fractures ( $11 \%$ versus $1 \%, p=0.020$ ). Moreover, age at SLE diagnosis, presence of osteoporosis and secondary hyperparathyroidism were the only variables significantly associated with a first fragility fracture in Cox Regression, with hazard ratios of $1.05(95 \% \mathrm{Cl} 1.02-1.08, \mathrm{p}=0.001), 2.84(95 \% \mathrm{Cl} 1.31-6.16, \mathrm{p}=0-008)$ and $4.49(95 \% \mathrm{Cl} 1.35$ 14.98, $p=0.015)$, respectively. When assessing treatment, a higher percentage of patients with fractures received osteoporotic treatment than those without fractures; bisphosphonate ( $79 \%$ versus $27 \%, p<0.001)$, denosumab ( $7 \%$ versus $0.5 \%, p=0.034)$, teriparatide $(7 \%$ versus $0 \%, p=0.012)$ and strontium ranelate $(7 \%$ versus $0 \%, p=0.12)$.

In assessing glucocorticoid dosing over time, we found no difference between patients that were diagnosed before and after 2001 (table 2). There was no statistically significant difference in average daily dosing or the proportion of patients treated with a higher dose of glucocorticoids. This was also true when we compared patients diagnosed before and after 2005 see supplementary table S1 material. As would be expected, the cumulative doses were increased in those diagnosed earlier as they have received treatment for a longer period.

We performed univariable COX regression to investigate the association between glucocorticoids and the onset of osteoporosis and osteopenia, as well as the development of fragility fractures. We calculated the hazard ratio of cumulative glucocorticoid dose, average daily dose, and treatment with glucocorticoids versus those not treated. There was no significant effect on the risk of fragility fractures (table 3), osteopenia (table 4) or osteoporosis (Table 5).

To investigate whether those patients on higher doses of glucocorticoids were more likely to be taking prophylactic bisphosphonates, we have calculated median daily steroid dose in patients taking bisphosphonates $(6.35 \mathrm{mg} /$ day $)$ and compared it with the daily steroid dose in patients not taking bisphosphonates $(6.25 \mathrm{mg} /$ day). There was no significant difference found $(p=0.684)$. Furthermore, the proportion of patients with an average daily dose of steroids $\geq 7.5 \mathrm{mg}$ that was taking bisphosphonates (36\%) did not differ significantly from the proportion of patients with a lower dose of steroids taking bisphosphonates (32\%) ( $p=0.539)$. Also, the proportion of patients with ever-high 
dose steroids (>20mg/day) that was taking bisphosphonates (38\%) did not differ significantly from the proportion of patients who never had a high dose and were taking bisphosphonates (32\%) $(p=0.441)$.

\section{Discussion}

Chronic glucocorticoid treatment is widely recognised as an important risk factor for the development of osteoporosis and fragility fractures. Due to the increased understanding of the harmful effects of glucocorticoids, we expected to see a change in glucocorticoid prescribing practice over the years. We were surprised to find no significant difference in glucocorticoid prescribing over the 28 years. Patients diagnosed before and after 2001 were both prescribed a low daily dose of around $6 \mathrm{mg} /$ day, so the majority of patients were treated with glucocorticoid doses $<7.5 \mathrm{mg} /$ day over 20 years prior to the publication of specific guidelines.

The requirement of a DEXA scan for analysis inclusion may have caused a bias in assessing glucocorticoid dosing in this cohort of patients. Higher glucocorticoid dosing, however, is an indication to perform a DEXA scan in our centre. So, it is most likely that this bias would have caused a selection of patients with higher glucocorticoid dosing further reinforcing the reassuring finding of a low average daily dose in our cohort.

When examining our cohort of 250 patients with SLE followed up over 28 years we did not find any statistically significant association with cumulative or average dosing of glucocorticoids and onset of osteoporosis or osteopenia, or development of fragility fractures. Also, there were no differences in the onset of osteoporosis or osteopenia, and development of fragility fractures, when comparing those treated with glucocorticoids and those who were not. The group not treated with glucocorticoids was small making up only $6 \%$ of the cohort and not a control group. Patients without DEXA scans were excluded from analysis so our cohort may not be reflective of all patients in the SLE clinic treated with glucocorticoids.

Data collection was retrospective which could contribute to an underdiagnosis of fragility fractures. A few patients with fragility fractures may have not have undergone DEXA scans or undergone DEXA scans in different centres so they were excluded from the analysis. Nonetheless, the number of patients with fragility fractures that did not undergo a DEXA scan is likely to be very small and our data incorporates a comprehensive selection of patients under follow-up in our clinic. We also included vertebral fractures in our definition of fragility fractures. These were identified from the patient record. As vertebral fractures are often asymptomatic and up to $46 \%$ may be missed on radiographs[14], there may be an underestimation of this diagnosis. 
Our findings are similar to previously reported cohorts. Fragility fractures occurred in $11.2 \%$ of our patients followed up for 4498 person-years (Figure 1 shows the cumulative risk of fracture over time). This is similar to the findings of the Ramsey-Goldman et al. study of a cohort of 702 SLE patients. They reported fracture occurrence of $12.3 \%$ on follow up of 5951 person-years[13]. In our cohort, osteoporosis was present in $16 \%$ of patients, whilst a recent systematic review reports a range of 1.4 - 68\% of osteoporosis in SLE cohort studies [15]. This provides reassuring data that the impact of selection bias on our cohort was probably small.

There are several possible explanations for our findings. The risk of osteoporosis or fracture associated with glucocorticoids is dependent on the dose and duration of treatment. The majority of papers report that daily dose is more significant than the cumulative dose $[1,16-18]$; conversely, some studies have found cumulative dose also to be an important risk factor[19]. A recent study of 16,000 Danish patients with fragility fractures receiving glucocorticoids, compared to controls, observed that patients with a high average daily dose, defined as $>15 \mathrm{mg} /$ day or a cumulative dose $>1 \mathrm{~g}$, were more likely to have a fragility fracture risk than those on a low dose $<7.5 \mathrm{mg} /$ day. There was no significant difference to those on the intermediate dose of $7.5-14.9 \mathrm{mg} /$ day. The results for low dose was associated with an odds ratio (OR) 1.37 for hip fractures, intermediate dose with OR 1.57 and high dose with OR 2.5. In those with cumulative dose $<1 \mathrm{~g}$ was associated with an OR 1.28 and those $\geq 1 \mathrm{~g}$ with an OR 1.64. The highest risk, however, was found in those with both high daily and cumulative dose, which is $>15 \mathrm{mg} /$ day and $>1 \mathrm{~g}$, with an OR of 2.94[20]. Despite this data, identifying the average daily dosage or cumulative dose of glucocorticoids at which the risk of osteoporosis or development of fragility fractures significantly increases remains elusive[21].

The median average daily glucocorticoid dose in our cohort was low at $6.20 \mathrm{mg} / \mathrm{day}$, and the average daily dose was not associated with low BMD, which is in agreement with other studies $[22,23]$. The mean cumulative dose, however, was high $>21 \mathrm{~g}$. reflective of the prolonged follow-up in our cohort of patients and maybe explaining the conflicting reports on the association of cumulative glucocorticoid dose and fracture risk[11]. Moreover, nearly all patients received prophylactic vitamin D supplementation and the majority were also treated with calcium supplementation. A Cochrane review found that two years of prophylactic vitamin $D$ and calcium treatment can improve the lumbar BMD by $2.6 \%$ in glucocorticoid-induced osteoporosis[24]. Moreover, a systematic review has confirmed the importance of treating low vitamin D in preventing osteoporosis in lupus patients[15]. It appears that the common treatment of our patients with vitamin $D$ and calcium ameliorated the negative impact of glucocorticoids. This is especially so since secondary hyperparathyroidism, which is the consequence of low total or activated vitamin $D$, was found to be one of the factors associated with increased risk of fragility fractures in this cohort. 
Our method for estimating glucocorticoid dosing was based on dosing documented by the physician in contemporaneous patient records. This is a relatively accurate method for measuring glucocorticoid dosing. Few trials have collected consecutive glucocorticoid dosing, instead of using more approximate estimations of dosing. Previous trials have examined glucocorticoid exposure versus no exposure; high, medium and low intensity treatment according to BILAG or approximated dosing based on the first ever steroid prescription $[11,18,20]$. We believe our data is a reasonably accurate reflection of prescribed and reported treatment. It is well documented, however, that non-adherence is an important issue in the management of SLE with $62 \%$ of patients reporting high adherence in the Lula study of 579 patients[25]. A meta-analysis of 11 studies reported a range of non-adherence of 4375\%. Assuming a significant level of nonadherence in our cohort would result in daily dosing of less than $6.20 \mathrm{mg} /$ day[26].

The cause of osteoporosis in lupus patients is multifactorial. There is increasing recognition of the impact of inflammation on bone remodelling, as the field of research into osteoimmunity evolves. The Hopkins lupus cohort of 1356 patients found significantly less osteoporosis and fewer fractures in patients with low disease activity or in clinical remission. The relationship with glucocorticoid dosing was not explored in this study. It may be that the patients in low disease activity or remission required reduced glucocorticoid dosing thereby lowering the proportion of patients developing osteoporosis[27]. Moreover, the impact of disease flares was also shown in a five-year longitudinal study from China[28]. They found that disease flares led to a decrease in bone density, as did glucocorticoids during follow-up. There was also an increase in bone mineral density in this cohort of patients when using anti-osteoporotic therapy. It is a weakness of our study that there was no reference to disease activity.

Our study had other limitations. Firstly, there was no matched control group, so we were unable to evaluate the impact of SLE disease course on BMD and fractures. Furthermore, there was some heterogeneity concerning the duration of follow up and the intervals between DEXA scans.

The use of statistical survival analysis, with Cox Regression, could compensate for the heterogeneity in the duration of follow-up. This kind of analysis takes into account the time when the event occurred (fracture or DEXA showing low BMD) and not only whether it occurred or not. This analysis also considers the subjects in whom the event did not occur during the time of follow-up (censored), thus reducing the risk of bias due to underestimating the occurrence of the event.

We report a substantial amount of data from a single centre large SLE cohort with a very long duration of follow up and an accurate estimation of longitudinal glucocorticoid dosing. We were pleased to confirm that our patients have been treated with low doses of glucocorticoids, with little difference 
between current and previous practice and, consistent with recently published guidelines. In our group of 250 patients, $94 \%$ of whom received glucocorticoids (together with common prescription of vitamin D and calcium), we found that glucocorticoid dosing was not associated with an increased risk of onset of osteoporosis or osteopenia, and with development of fragility fractures. Although this is a retrospective analysis of a large SLE cohort that provides interesting observations, a prospective study including more patients not on glucocorticoids would provide more robust data to underpin these observations and confirm their validity. 
Table 1: Characteristics of the population studied and comparison between patients with and without fragility fractures.

\begin{tabular}{|c|c|c|c|c|c|}
\hline & & Total & Without fracture & With fracture & $\mathbf{P}$ \\
\hline \multicolumn{2}{|l|}{ Total, N } & 250 & 222 & 28 & \\
\hline \multicolumn{2}{|c|}{ Females, $\mathrm{N}(\%)$} & $229(92)$ & $203(91)$ & $26(93)$ & 1.000 \\
\hline \multirow[t]{4}{*}{ Ethnicity } & Caucasian, N (\%) & $130(52)$ & $114(52)$ & $16(57)$ & \multirow{4}{*}{0.569} \\
\hline & Afro-Caribbean, N (\%) & $62(25)$ & $56(25)$ & $6(21)$ & \\
\hline & South Asian, N (\%) & $45(18)$ & $39(18)$ & $6(21)$ & \\
\hline & Other, N (\%) & $12(5)$ & $12(5)$ & $0(0)$ & \\
\hline \multicolumn{2}{|c|}{ Age SLE diagnosis(y), median (IQR) } & $27(20)$ & $27(18)$ & $36(16)$ & 0.009 \\
\hline \multicolumn{2}{|c|}{ Time follow-up(y), median (IQR) } & $17(10)$ & $17(9)$ & $22(11)$ & 0.001 \\
\hline \multicolumn{2}{|c|}{ DEXA osteoporosis, N (\%) } & $40(16)$ & $30(14)$ & $10(36)$ & 0.006 \\
\hline \multicolumn{2}{|c|}{ Age at $1^{\text {st }}$ fracture $(y)$, mean \pm SD } & N/A & N/A & $51 \pm 16$ & N/A \\
\hline \multicolumn{2}{|c|}{ Ever treatment with glucocorticoids, $\mathrm{N}(\%)$} & $235(94)$ & 209 (94) & $26(93)$ & 0.678 \\
\hline \multicolumn{2}{|c|}{ Average daily dose PDN (mg), median (IQR) } & $6.20(3.13)$ & $6.23(3.16)$ & $5.36(3.40)$ & 0.127 \\
\hline \multicolumn{2}{|c|}{ Average daily dose PDN $\geq 7.5 \mathrm{mg}, \mathrm{N}(\%)$} & $79(32)$ & $72(32)$ & $7(25)$ & 0.425 \\
\hline \multicolumn{2}{|c|}{ Cumulative dose PDN(g), median (IQR) } & $21.68(22.03)$ & $20.96(21.08)$ & $25.19(20.43)$ & 0.229 \\
\hline \multicolumn{2}{|c|}{ Body mass index, median (IQR) } & $24(6)$ & $24(6)$ & $23(6)$ & 0.440 \\
\hline \multicolumn{2}{|c|}{ Smokers, N (\%) } & $34(27)$ & $29(26)$ & $5(33)$ & 0.385 \\
\hline \multicolumn{2}{|c|}{ Vitamin D deficiency, N (\%) } & $27(11)$ & $22(10)$ & $5(18)$ & 0.201 \\
\hline \multicolumn{2}{|c|}{ Vitamin D supplement, N (\%) } & $232(93)$ & $204(92)$ & $28(100)$ & 0.236 \\
\hline \multicolumn{2}{|c|}{ Calcium supplement, N (\%) } & $212(85)$ & $186(84)$ & $26(93)$ & 0.272 \\
\hline \multicolumn{2}{|c|}{ Hyperparathyroidism, N (\%) } & $6(2)$ & $3(1)$ & $3(11)$ & 0.020 \\
\hline \multicolumn{2}{|c|}{ ESRD, N (\%) } & $8(3)$ & $8(4)$ & $0(0)$ & 0.603 \\
\hline \multicolumn{2}{|c|}{ Hyperthyroidism, N (\%) } & $3(1)$ & $3(1)$ & $0(0)$ & 1.000 \\
\hline \multicolumn{2}{|c|}{ Gonadal failure, N (\%) } & $4(2)$ & $2(1)$ & $2(7)$ & 0.063 \\
\hline \multicolumn{2}{|c|}{ Rheumatoid arthritis, N (\%) } & $12(5)$ & $9(4)$ & $3(11)$ & 0.139 \\
\hline \multicolumn{6}{|c|}{ Anti-osteoporotic treatment (ever) } \\
\hline \multicolumn{2}{|c|}{ Bisphosphonates, N (\%) } & $82(33)$ & $60(27)$ & $22(79)$ & $<0.001$ \\
\hline \multicolumn{2}{|c|}{ Denosumab, N (\%) } & $3(1)$ & $1(0.5)$ & $2(7)$ & 0.034 \\
\hline \multicolumn{2}{|c|}{ Teriparatide, $\mathrm{N}(\%)$} & $2(1)$ & $0(0)$ & $2(7)$ & 0.012 \\
\hline \multicolumn{2}{|c|}{ Strontium ranelate, $\mathrm{N}(\%)$} & $2(1)$ & $0(0)$ & $2(7)$ & 0.012 \\
\hline \multicolumn{2}{|c|}{ Raloxifene, N (\%) } & $2(1)$ & $1(0.5)$ & $1(4)$ & 0.212 \\
\hline \multicolumn{2}{|c|}{ Hormonal replacement therapy, $\mathrm{N}(\%)$} & $4(2)$ & $3(1)$ & $1(4)$ & 0.380 \\
\hline
\end{tabular}

SLE: Systemic lupus erythematosus; PDN: prednisolone; ESRD: End-stage renal disease 
Table 2: Comparison between patients diagnosed before or after 2001

\begin{tabular}{|c|c|c|c|}
\hline & Year diagnosis $<2001$ & Year diagnosis $\geq 2001$ & $\mathbf{P}$ \\
\hline $\mathbf{N}$ & 133 & 117 & \\
\hline Time follow-up(y), median (IQR) & $22(6)$ & $12(5)$ & $<0.001$ \\
\hline Cumulative dose PDN(g), median (IQR) & $24.5(22.5)$ & $19.2(19.7)$ & 0.017 \\
\hline Average daily dose $\mathrm{PDN}(\mathrm{mg})$, median (IQR) & $6.1(2.8)$ & $6.3(3.1)$ & 0.068 \\
\hline Average daily dose PDN $\geq 7.5 \mathrm{mg}, \mathrm{N}(\%)$ & $36(27)$ & $43(37)$ & 0.100 \\
\hline Ever treatment with steroids, $\mathrm{N}(\%)$ & $125(94)$ & $110(94)$ & 0.991 \\
\hline
\end{tabular}

Table 3: Effect of glucocorticoids in the risk of fragility fractures

\begin{tabular}{lccc}
\hline Univariable COX Regression & B coefficient & HR [95\%Cl] & p \\
\hline Cumulative dose & 0.000 & $1.000[1.000-1.000]$ & 0.963 \\
Average daily dose & 0.024 & $1.024[0.923-1.137]$ & 0.651 \\
Ever treatment with steroids & 0.429 & $1.536[0.359-6.579]$ & 0.563 \\
\hline
\end{tabular}

HR: hazard ratio

Table 4: Effect of glucocorticoids in the risk of osteopenia

\begin{tabular}{lccc}
\hline Univariable COX Regression & B coefficient & HR [95\%Cl] & p \\
\hline Cumulative dose & 0.000 & $1.000[1.000-1.000]$ & 0.030 \\
Average daily dose & 0.002 & $1.002[0.956-1.049]$ & 0.948 \\
Average daily dose PDN $\geq 7.5 \mathrm{mg}$ & -0.072 & $0.930[0.649-1.333]$ & 0.694 \\
Ever treatment with steroids & 0.063 & $1.065[0.541-2.096]$ & 0.855 \\
\hline HR: hazard ratio & & &
\end{tabular}

HR: hazard ratio

Table 5: Effect of glucocorticoids in the risk of osteoporosis

\begin{tabular}{lccc}
\hline Univariable COX Regression & B coefficient & HR [95\%Cl] & $\mathbf{p}$ \\
\hline Cumulative dose & 0.000 & $1.000[1.000-1.000]$ & 0.910 \\
Average daily dose & 0.020 & $1.020[0.935-1.113]$ & 0.652 \\
Average daily dose PDN $\geq 7.5 \mathrm{mg}$ & 0.271 & $1.312[0.684-2.516]$ & 0.415 \\
Ever treatment with steroids & -0.205 & $0.815[0.251-2.645]$ & 0.733 \\
\hline
\end{tabular}

HR: hazard ratio 


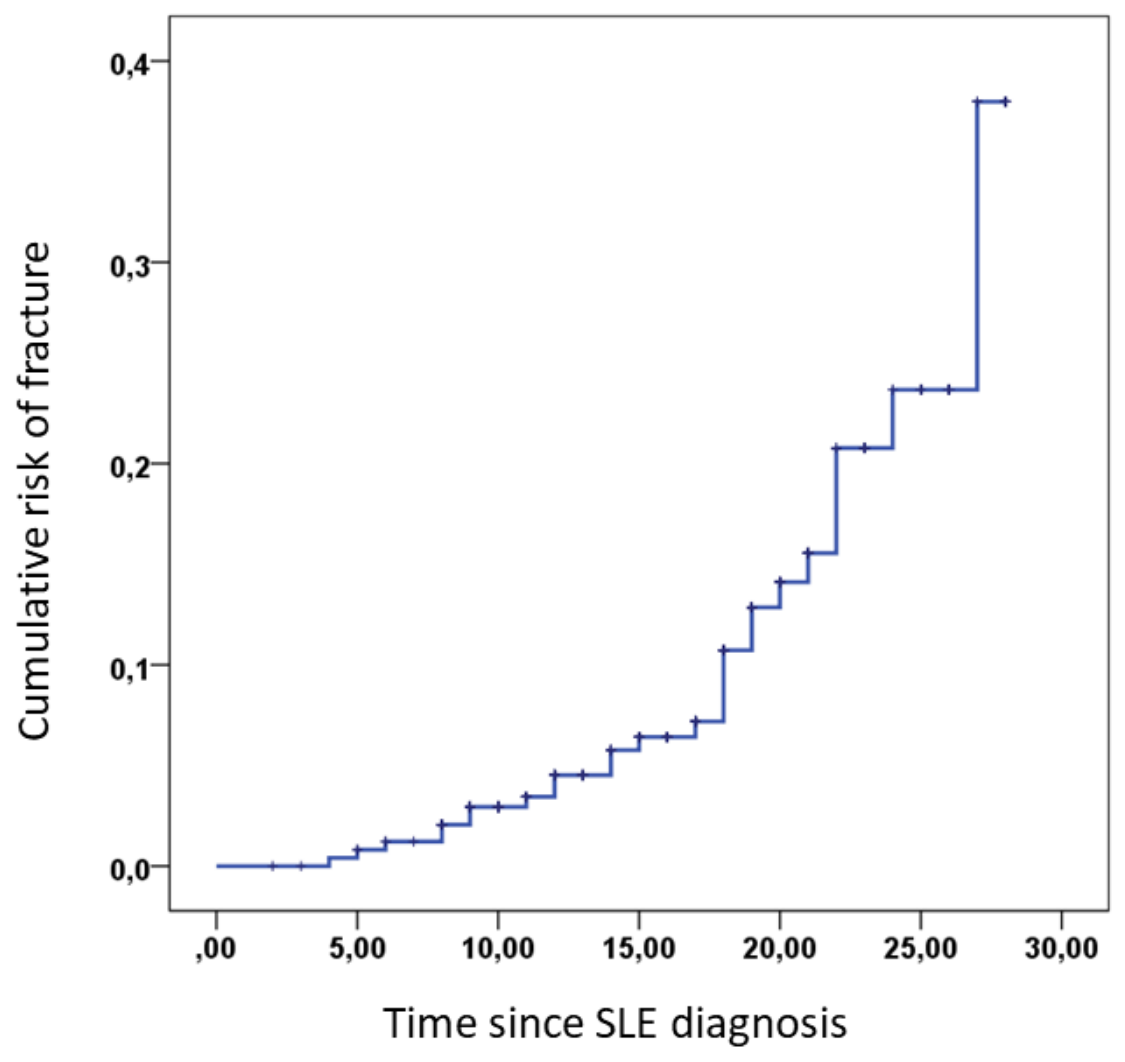

$\begin{array}{llllllll}\text { N at risk } & 250 & 247 & 215 & 154 & 80 & 32 & 0\end{array}$

Figure 1: Kaplan-Meier curve showing cumulative risk for the first fragility fracture in our group of 250 patients with SLE. 
Bibliography

1. van Staa TP, Leufkens HG, Abenhaim L, Zhang B, Cooper C. Oral corticosteroids and fracture risk: relationship to daily and cumulative doses. Rheumatology 2000;39:1383-1389.

2. Adami G, Saag KG. Glucocorticoid-induced osteoporosis: 2019 concise clinical review. Osteoporos. Int. 2019;30:1145-1156.

3. Compston J. Glucocorticoid-induced osteoporosis: an update. Endocrine 2018;61:7-16.

4. Hardy RS, Zhou H, Seibel MJ, Cooper MS. Glucocorticoids and bone: consequences of endogenous and exogenous excess and replacement therapy. Endocr. Rev. 2018;39:519-548.

5. Fanouriakis A, Kostopoulou M, Alunno A, Aringer M, Bajema I, Boletis JN, et al. 2019 update of the EULAR recommendations for the management of systemic lupus erythematosus. Ann. Rheum. Dis. 2019;78:736-745.

6. Al Sawah S, Zhang X, Zhu B, Magder LS, Foster SA, likuni N, et al. Effect of corticosteroid use by dose on the risk of developing organ damage over time in systemic lupus erythematosus-the Hopkins Lupus Cohort. Lupus Sci. Med. 2015;2:e000066.

7. IMAJ | The Israel Medicine Association Journal | Volume 11, Number 8, August 2009 | Osteoporosis in Patients with Systemic Lupus Erythematosus [Internet]. [cited 2019 Oct 6];Available from: https://www.ima.org.il/MedicineIMAJ/viewarticle.aspx?year=2009\&month=08\&page=486

8. Sun $Y-N$, Feng $X-Y, H e ~ L$, Zeng $L-X, H a o ~ Z-M, ~ L v ~ X-H$, et al. Prevalence and possible risk factors of low bone mineral density in untreated female patients with systemic lupus erythematosus. Biomed Res. Int. 2015;2015:510514.

9. Mendoza-Pinto C, García-Carrasco M, Sandoval-Cruz H, Escárcega RO, Jiménez-Hernández M, Etchegaray-Morales I, et al. Risks factors for low bone mineral density in pre-menopausal Mexican women with systemic lupus erythematosus. Clin. Rheumatol. 2009;28:65-70.

10. Lai EL, Huang WN, Chen HH, Hsu CY, Chen DY, Hsieh TY, et al. Ten-year fracture risk by FRAX and osteoporotic fractures in patients with systemic autoimmune diseases. Lupus 2019;28:945-953.

11. Bultink IEM, Harvey NC, Lalmohamed A, Cooper C, Lems WF, van Staa TP, et al. Elevated risk of clinical fractures and associated risk factors in patients with systemic lupus erythematosus versus matched controls: a population-based study in the United Kingdom. Osteoporos. Int. 2014;25:1275-1283.

12. Tedeschi SK, Kim SC, Guan H, Grossman JM, Costenbader KH. Comparative fracture risks among united states medicaid enrollees with and those without systemic lupus erythematosus. Arthritis Rheumatol. 2019;71:1141-1146.

13. Ramsey-Goldman R, Dunn JE, Huang CF, Dunlop D, Rairie JE, Fitzgerald S, et al. Frequency of fractures in women with systemic lupus erythematosus: comparison with United States population data. Arthritis Rheum. 1999;42:882-890.

14. Lems WF. Clinical relevance of vertebral fractures. Ann. Rheum. Dis. 2007;66:2-4. 
15. Salman-Monte TC, Torrente-Segarra V, Vega-Vidal AL, Corzo P, Castro-Dominguez F, Ojeda F, et al. Bone mineral density and vitamin D status in systemic lupus erythematosus (SLE): A systematic review. Autoimmun. Rev. 2017;16:1155-1159.

16. van Staa TP, Leufkens HGM, Cooper C. The epidemiology of corticosteroid-induced osteoporosis: a meta-analysis. Osteoporos. Int. 2002;13:777-787.

17. Van Staa TP, Laan RF, Barton IP, Cohen S, Reid DM, Cooper C. Bone density threshold and other predictors of vertebral fracture in patients receiving oral glucocorticoid therapy. Arthritis Rheum. 2003;48:3224-3229.

18. Amiche MA, Albaum JM, Tadrous M, Pechlivanoglou P, Lévesque LE, Adachi JD, et al. Fracture risk in oral glucocorticoid users: a Bayesian meta-regression leveraging control arms of osteoporosis clinical trials. Osteoporos. Int. 2016;27:1709-1718.

19. Balasubramanian A, Wade SW, Adler RA, Lin CJF, Maricic M, O'Malley CD, et al. Glucocorticoid exposure and fracture risk in patients with new-onset rheumatoid arthritis. Osteoporos. Int. 2016;27:3239-3249.

20. Amiche MA, Abtahi S, Driessen JHM, Vestergaard P, de Vries F, Cadarette SM, et al. Impact of cumulative exposure to high-dose oral glucocorticoids on fracture risk in Denmark: a population-based case-control study. Arch. Osteoporos. 2018;13:30.

21. Grossman JM, Gordon R, Ranganath VK, Deal C, Caplan L, Chen W, et al. American College of Rheumatology 2010 recommendations for the prevention and treatment of glucocorticoidinduced osteoporosis. Arthritis Care Res. (Hoboken) 2010;62:1515-1526.

22. Sambrook PN, Eisman JA, Yeates MG, Pocock NA, Eberl S, Champion GD. Osteoporosis in rheumatoid arthritis: safety of low dose corticosteroids. Ann. Rheum. Dis. 1986;45:950-953.

23. Sambrook PN, Cohen ML, Eisman JA, Pocock NA, Champion GD, Yeates MG. Effects of low dose corticosteroids on bone mass in rheumatoid arthritis: a longitudinal study. Ann. Rheum. Dis. $1989 ; 48: 535-538$.

24. Homik J, Suarez-Almazor ME, Shea B, Cranney A, Wells G, Tugwell P. Calcium and vitamin D for corticosteroid-induced osteoporosis. Cochrane Database Syst. Rev. 2000;:CD000952.

25. Chehab G, Sauer GM, Richter JG, Brinks R, Willers R, Fischer-Betz R, et al. Medical adherence in patients with systemic lupus erythematosus in Germany: predictors and reasons for nonadherence - a cross-sectional analysis of the LuLa-cohort. Lupus 2018;27:1652-1660.

26. Mehat P, Atiquzzaman M, Esdaile JM, AviÑa-Zubieta A, De Vera MA. Medication nonadherence in systemic lupus erythematosus: A systematic review. Arthritis Care Res. (Hoboken) 2017;69:1706-1713.

27. Petri M, Magder LS. Comparison of remission and lupus low disease activity state in damage prevention in a united states systemic lupus erythematosus cohort. Arthritis Rheumatol. 2018;70:1790-1795.

28. Zhu TY, Griffith JF, Au S-K, Tang X-L, Kwok AW, Leung P-C, et al. Bone mineral density change in systemic lupus erythematosus: a 5-year followup study. J. Rheumatol. 2014;41:1990-1997. 